\title{
Adult Grade III Lymphomatoid Granulomatosis
}

National Cancer Institute

\section{Source}

National Cancer Institute. Adult Grade III Lymphomatoid Granulomatosis. NCI

Thesaurus. Code C115150.

Grade III lymphomatoid granulomatosis that occurs in adulthood. 